\title{
From Context to Competencies
}

\author{
Ira Diethelm and Christina Dörge \\ University of Oldenburg, Computer Science Education, \\ 26111 Oldenburg, Germany \\ \{Ira.Diethelm, Christina.Doerge\} @informatik.uni-oldenburg.de
}

\begin{abstract}
For classes in informatics ${ }^{1}$ it gets more and more important to develop a sustainable curriculum because computer technology and its related items are subject to frequent changes. This leads to the problem that the content of informatics courses suffers from fast decay time. And to make things worse: What we are teaching our pupils and students is far from sustainable knowledge. The scientific community has reacted on that by developing concepts like "key skills" and "competencies". But what does this mean for a teacher or lecturer? How can those skills be acquired by courses? In which way has the course to be constructed to meet these means? This paper discusses the relationship between educational standards in informatics and the development of teaching units to meet them. The concept of "context" will be introduced and used to show a way to plan courses by a context related approach on the one hand and to give an idea on how to find a different way to develop educational standards, how to improve and how to evaluate them on the other hand.
\end{abstract}

Keywords: Educational standards, Informatics in Context, competencies, key skills, Informatics education.

\section{Introduction}

Due to the changing situation in society, it became important to be familiar with some fundamental facts of informatics: Information technology and computers are a concept which is connected to every part of our life. Therefore, a general education of these topics is an important goal for schools and universities. But computer technology is a fast changing subject: New machines, programs and ideas pop up nearly every day. So it becomes hard for teachers and lecturers to develop a concept which helps them to educate their pupils and students for the demands of their future lives. "Life long learning" is the new buzz word for this problematic and the ideas of "key skills" and "competencies" were developed. But what does this mean? Do teachers have to get away from content-based curricula? How can we arrange teaching units to equip our students with the required "competencies", which enable them to adapt to the rapid changes specific for informatics?

The important questions for the development of a suitable curriculum are:

- What are the "important competencies" and which ones are more and which one are less important?

\footnotetext{
${ }^{1}$ Used in this paper as a summary for computer science, IT, ICT, etc., like in [8].
} 
- How can be assured to cover all important "competencies"?

- What kind of content could be used to teach "competencies"?

- How can a system for the development of teaching units related to certain competencies be created?

- How can competencies be achieved in a motivating way?

In this paper, we will first give a short insight in the competency debate, the concept of educational standards, and how educational standards are created. In addition, we will present a different idea to develop educational standards in informatics which mostly orients on teaching practice - which is a context oriented approach. The context oriented approach for teaching informatics competencies will also be introduced in this paper. The conclusion will give a summarized overview.

\section{Brief Overview on the Competency Debate}

The debate on "key qualifications" and competencies was raised in Germany in 1974 by Dieter Mertens. His paper was a reaction to a change of the job market: Students were not well prepared for the needs of their job. Additionally, the students' education was not covering the demands to do a specific job for a whole life. "Life long learning" became a request due to the constantly changing job descriptions (see Mertens, [7]). He called his concept "key qualifications".

A lot of papers about competencies and key qualifications were published in the ongoing years after the "PISA-shock". The results especially of German students were, against all national expectations, below the international average in all three domains of the PISA test in 2001 (see [11], p. 147). By the end of the 1990s, in these publications the terms "qualification" and "competencies" were used so often, that it became nearly impossible to distinct between the different definitions and concepts: Some scientists used the same terms but different concepts and others the same concept with different terms. Some others were not giving a concept or definition at all. The whole discussion about "key qualifications" and "competencies" became a new updraft in Europe when the Bologna-Process started.

At that point a lot of scientists have discussed on what competencies are and if they are measurable or not. At the end - if it can be described in that way - the OECD (in co-operation with the UNESCO) gave a definition on what key competencies are in their report in 2005. They offered a concept of competencies, which included measurability. This was important, if competencies should be a concept of use for educational standards where the results of learning should be able to be evaluated (see [9], p. 8 and p. 10ff). A more detailed overview on that topic can be found in (see Dörge, [3]).

\section{The Development of Educational Standards}

Often the concepts of competencies are connected to educational standards. An educational standard itself can consist of many different types: There are content standards / curriculum standards, opportunity-to-learn-standards, performance standards and output-oriented standards. Performance standards are divided in three categories or levels, which are separated by the expected results: The first level is a minimal 
standard which means, that at very student of a certain age should have the required knowledge as a minimal level. Another level is the "norm" and means that the average student has the described knowledge (not necessarily every student). The last level means, that only the best students have the described knowledge. The outputoriented standards are based on competencies: Competencies are the "product" which will be measured at a certain age.

\subsection{The Regular Way to Create Educational Standards}

Usually, educational standards are created in a normative way by a group of some professionals which are working for a higher commission like the ACM, NCTM (National Council of Teachers of Mathematics), the German Kultusminister-Konferenz (KMK, Conference of the Ministers of Education) or other communities. Then, the outcome of this commission is somehow presented to the public. After some cycles of discussion, where input from the basis is followed to a certain degree, the educational standard is passed through some administration levels. Only some countries involve empirical evaluation in this process (e.g. in Switzerland, see [11], p. 277ff). In most countries research on this topic is postponed after publishing them and only used to evaluate if the educational standards are suitable. But it takes a lot of time until the results of this research is reflected and integrated in new versions of the standards.

Due to the fact that current educational standards focus on competencies, instead of teaching content, the lists of competencies are published in a context free way. Although professionals in these commissions ${ }^{2}$ mostly think of possible teaching context

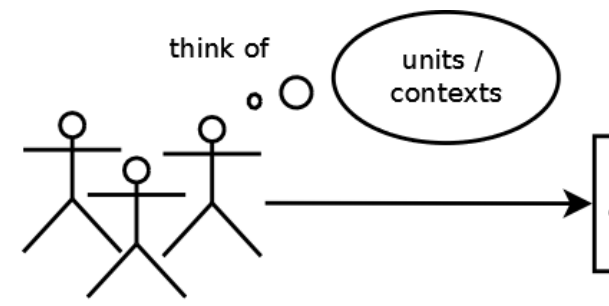

members of commission

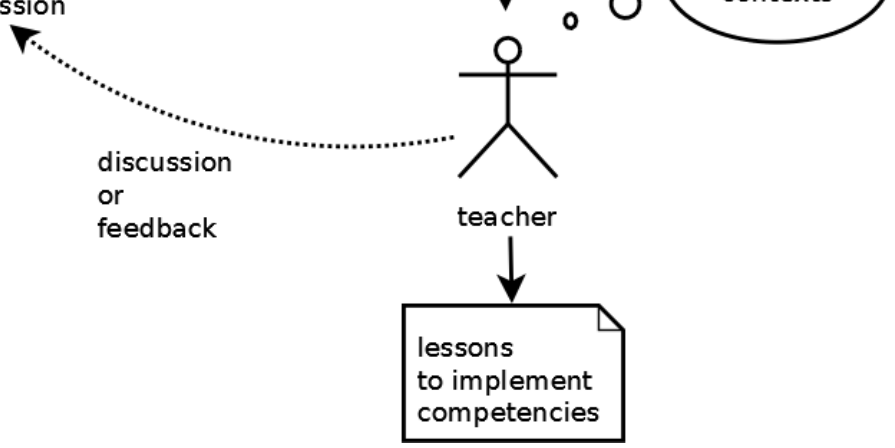

Fig. 1. The top-down process of creating lessons from educational standards

\footnotetext{
${ }^{2}$ Note: One of the authors of this paper has been part of such commissions.
} 
for these competencies, it is common not to record them. This comes with some obstacles: Teachers have to develop didactical concepts and plan how to arrange teaching units to achieve these competencies. They need to decide what the right contents are, see Fig. 1. It would probably be more efficient if they knew the contexts the commissions thought about. These thoughts could support the teachers developing their lessons.

As an example we'll have a closer look at the educational standards for informatics next.

\subsection{The Educational Standards for Informatics Lessons in Germany}

Which competencies should be taught in informatics lessons? The "Gesellschaft für Informatik (GI, German Association for Informatics)" answered this question by developing educational standards (see GI, [4]) published in 2008. These GI-standards were meant as a recommendation for the German Government. The main difference between these standards and those of the German ministries of education is that the GI-standards are minimal standards instead of average ones.

The competencies of the minimal GI-standards are divided into two sections: The "Content sections", which are "content-related", and the "Process sections", where the competencies are "process-related". Both sections are not separated but interlocked with each other: Process-related competencies are gained with content. Without content it would not be specifically informatics (see GI, [4] p. 45). The structure of the GI-standard was derived from the US-standard NCTM from 2000 (p.2). For an English description of the GI-standards see [1].

To ease the problems creating lessons for competencies and thinking of a suitable context to reach them, the GI added some more or less detailed examples for teaching units. But often the problem stays like it was: First the experts have to discuss if the one or the other competency fits in the educational standard and then again they have to discuss about an example how to teach it, like displayed in Fig. 1. And also it may lead to the problem that teachers focus on single competencies and do not see the connections between many different sections of competencies in informatics. This is why we suggest another way of creating educational standards in informatics in the next section.

\section{A New Approach in Designing Educational Standards}

The most common way for setting up educational standards is done by a set of professionals or representatives of the ministry of education who develop standards in a normative way like described above in section 3 and Fig. 1. The teachers have to bring those competencies to life by preparing lessons and setting up suitable teaching units. This approach can be called "competencies-to-lessons". But it takes a lot of time to establish a tradition of teaching new standards and to create suitable teaching units that motivate students. To motivate students you have to teach in a context that relates to their own everyday life. In the field of informatics these topics change so fast that there is not enough time for creating a tradition. 
If we would use topics from the last decade we would annoy our students or would support the misjudgment that informatics at school has nothing to do with their life and future. This happens in classes where students are still forced to program tiny functions or games that their mobile phones do better. Other ways of creating standards and lessons for informatics are needed. One of this other ways could be called “context-to-competencies", and will be described as follows and in Fig. 2.

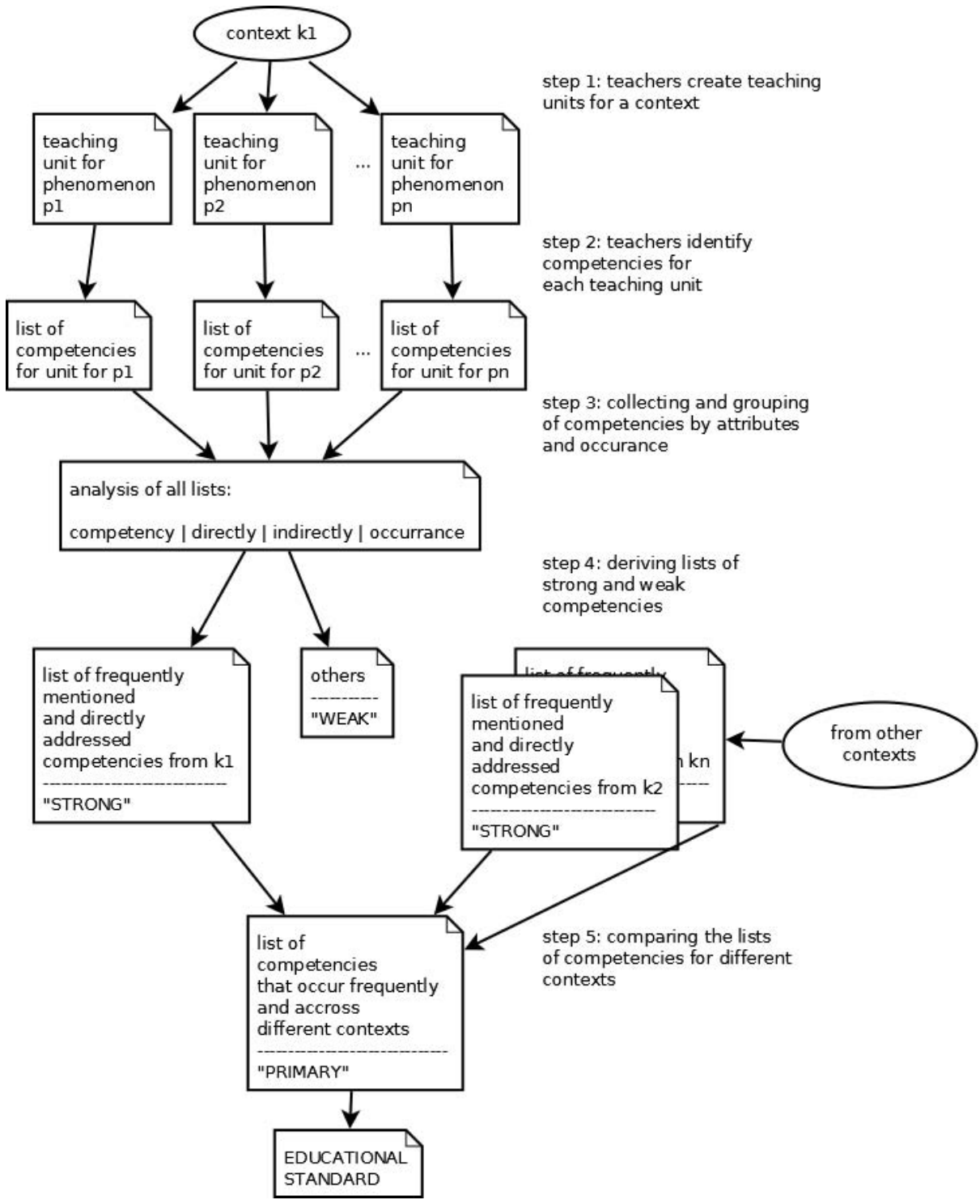

Fig. 2. Model for deriving educational standards from contexts 


\subsection{From Context to Competencies}

For this approach a set of important contexts is derived from literature, observation of every day life and empirical research about the most important phenomena and contexts that relate to informatics. For example we name them $k_{1}, \ldots, k_{m}$ and $p_{1}, \ldots, p_{n}$, where many phenomena belong to one context $k$ and define this context. It is also possible that a few phenomena belong to more than one context. The contexts are not disjoint regarding their phenomena.

In the first step of this approach, teachers create teaching units for these phenomena and nominate competencies $c_{1}, \ldots, c_{p}$ that should be gained with these units within a given context in the second step. As a result we get a set of competencies $c$ for each teaching unit for a context $k$ as a suggestion from the teachers, see also Fig 2. The competencies will also have to be marked according to whether they are addressed directly or indirectly by that unit.

In the third step of this process we collect all competencies that belong to a given context $k$. Then all those competencies that may be gained by this given context are merged and classified if they are addressing the mentioned competencies directly or indirectly and how often they occur in different teaching units. Comparing them the following result will be expected: Some competencies are mentioned more often than others, some are addressed directly and some are gained indirectly during the set of lessons for a context. And some are context related and some are not. With this, the competencies can be divided in "strong" and "weak" competencies in the next step.

This process has to be repeated for every other context $\mathrm{k}$. Comparing the lists of strong competencies there will be some that occur across different contexts and therefore are named "primary competencies". These competencies can now be listed in an educational standard together with the teaching units mentioned as a suggestion. We will illustrate this with an example unit in section 5.1.

Over the course of time new teaching units for changing contexts will have to be created. But we assume that after the analysis of these new units the "primary competencies" will be found there as well. So we will get teaching units that address longlasting competencies which will fit in our fast changing world. To emphasize this idea, we will now discuss a time-independent context which is representative and not only related to informatics.

\subsection{Example: Writing Text}

Let's take a closer look at three ways of writing a text. The first way is the oldfashioned way - writing with a pen on paper. The second way is done with a typewriter on a piece of paper and the third one with a computer using a word processing system. Even though the medium has changed, the competencies for writing texts remain the same: The writer has to have the competency of designing a text, with a structure and the skill to use the words correctly and to give the text a meaning. In addition to these basic skills, other competencies are necessary to use and understand the medium correctly. For example:

1. How is a pen used on paper - without scratching the paper without dropping ink on the paper, without smudging the already written letters? 
2. How is a typewriter used? Included are competencies on how to type (e.g. 10-fingers-system) and to push down the types in that way, that all letters have the same density and the text is typed with an acceptable speed.

3. How is the computer used with its specific applications (the operating system, the word-processing system) to get the text ready and how is it printed out?

Due to the fact that software and hardware is fast-changing it is not appropriate to teach only competencies how to use a specific medium, it is necessary to prepare students for life-long-learning and to teach long-lasting competencies. The focus of informatics lessons is to make students understand the basic functions make them able to reflect and to cope with occurring phenomena during the use of information processing.

One thought behind all this might be tempting: Maybe, the context special competency has been gained with is changing during time. But this is not affecting the competencies required: If the idea is to write a letter we still have to teach our students the concept of a letter and what the structure of a letter is and how it can be written, to be official or romantic (this might be part of e.g. English lessons) - even so the program has changed, we are working with.

For informatics lessons this could mean: Even when the word-processing programs change during time - maybe from an older version of Mircosoft Word to the current one: The text still has to be formatted, saved, printed etc. And the word processing system won't have changed on the conceptual level. If we teach the concepts behind the programs and connect them to their phenomena of an actual context, we give our students something they could rely on for a lifetime.

Therefore, a better approach for designing educational standards could be to collect big amounts of contexts and competencies gained from these contexts like described above. This could lead to a set of tools which teachers could really use: competencies and the combination of contexts, from which they could learn from. To give a closer insight an overview on the context based approach and its roots will be provided in the next section.

\section{What Is Informatics in Context?}

The definition of competency given by Franz E. Weinert is not only based on cognitive understandings and abilities, but also on motivation and willingness to apply these to different problems and on interaction with other people (see [12]). As a result of this, students' motivation and the application of knowledge are not something that begins after the development of competency; they are parts of competencies and have to be considered already in the learning process (see [10]).

Context-oriented approaches for schools have been established in Germany, the UK, and other countries after the PISA-shock to achieve a higher motivation for natural sciences and to support the vocational decisions of students. The largest project, "Chemistry in Context - ChiK", has been implemented in schools since 2002. ChiK aims at the change of teaching practice for more "authentic science" in classes and thus make it more interesting for students (see e.g. Parchmann, [10]). Therefore, 
teaching units have to be based on relevant contexts. These contexts should not only be used as a motivation at the beginning but also be present in all following parts of the teaching unit. ChiK-units aim to raise the variation of teaching methods and to point out basic concepts (like the relationship between behavior and structure). ChiK provides a pattern with four phases per unit (see [10] or [2]):

1. Phase of contact (e.g. a question or debate): personal relevance, interest;

2. Phase of curiosity and planning (e.g. a mind map): identify important questions;

3. Phase of elaboration: inquiry, results, presentation;

4. Phase of deepening and connecting: reflection, understanding, personal relevance.

Koubek et al. suggested an approach of Informatics in Context which is called IniK, abbreviated from the German title Informatik im Kontext, which is derived from the other context projects (see [6]). IniK has three aims:

1. orientation on relevant contexts;

2. variety of teaching methods and

3. principles and standards.

Several quite usable teaching units for this approach can already be found in [5]. One of them covers the context of "file sharing". In the following part we show how this unit can be analyzed to identify competencies in the way described above.

\subsection{Example for Informatics in Context: File Sharing}

"File sharing" is a topic every student nowadays already came or will come across. Therefore this fits the requirement to choose a context which relates to the world of the students. Strongly connected to "file sharing" is the concept of copyright. "File sharing" is part of computer network technology but also a legal and moral problem in the Internet. There are several ways to make the topic "file sharing" interesting. For our approach we are going to describe four phases separately:

1. The Entry Phase

2. The Working Phase

3. The Immersion and Linking Phase

4. The Knowledge Assessment Phase

\subsubsection{The Entry Phase}

There are many different possible ways to enter this context. A first impulse could be given by writing the words "pirate copy" at the black board. Another way could be done by starting a discussion with a sentence like "I have heard that a student of a different (or this) school has been questioned by the police for sharing music files online". There are also many current articles from newspapers, journals, TV shows etc. which can be used. These are the phenomena $p$ belonging to this context. 
After this impulse the students have to discuss their perspective and their experiences with this topic. They state questions on pieces of paper for the lessons and sort them (Note: These questions will be the guide for the lessons). And now, goals have to be set about the outcome (possible outcomes: poster for the school, presentation for the class or parents) and working groups have to be formed.

Questions that students would state and answer later on could be as follows:

- How differs a pirate copy from a normal copy and how can they be distinguished?

- What is copyright, copyleft, freeware, shareware, GPL etc.?

- When is copying (a CD, DVD, file, program) legal or illegal?

- What does a file sharing program do?

- How comes that the police knows my address if they only had my IP?

- What kind of hints to my identity do I leave in the Internet while surfing?

- $\quad \ldots$

\subsubsection{The Working Phase}

In working groups the students discuss their questions. The main aspect of the teachers work is to advise and NOT TO GUIDE. He / She can help to clear questions about the correctness and rightness of the answers they are looking for and he / she is preparing / providing the needed materials. The students make notes for their presentation and create their posters.

\subsubsection{Immersion and Linking Phase}

The students present their results in class and discuss these together. A lawyer could review the results of the class, if there is the possibility. The students discuss how to present the results to the whole school (e.g. in the format of an information event) and / or their parents and plan it.

\subsubsection{The Knowledge Assessment Phase}

Possible questions to control and assess the gained competencies about the topic could be like the following examples:

a. Explicate the way of data from the Net to your own PC when starting a download. (Draw a sketch from starting point of the data until the point of saving it on your hard drive)

b. Sort the necessary steps and technical parts of (a) by using the three-layermodel

c. Read the comic / the story (provided by the teacher). Describe the content of the comic / story with your own words

d. Explicate how the law was violated in (c) by the main character and by the other persons of this particular comic / story. Also explain what should have been different to assure that no law was broken.

A last possible step to conclude the experiences made above could be "decontextualization" (adopting the concept to other content), e.g. comparing the concept of file sharing with the streaming technology used by web-TV sites. 


\subsection{Possibly Gained Competencies}

With a description of a teaching unit for "informatics in context" like the one above or even more detailed it is possible to discuss the competencies that may be gained using it. According to the fact that especially the process related competencies differ according to the teaching methodology the teacher uses and every class behaves a bit different, the list of competencies that are gained with a teaching unit differs also. It would be an important advancement in research if we had an approach to reliably derive the most important competencies from a teaching unit. Until then we have to work with examples like the one from section 5.1.

Let's assume that a big enough set of teachers thought about this unit for the context "file sharing" $(k)$ and gave a list of possibly gained competencies each, (the direct and the indirect ones as introduced in section 4). These lists could be analyzed for the competencies mentioned most frequently for this context. These are the competencies $c$ directly addressed.

The following list shows some example competencies, which could have been mentioned. The students are able to...

- $\quad \mathrm{c}_{1}$; characterize hardware and software components

- $\mathrm{c}_{2}$ : illustrate the function of P2P-systems with graphical models

- $c_{3}$ : distinguish different license models like GPL or shareware

- $\mathrm{c}_{4}$ : plan group work

- $\mathrm{c}_{5}$ : cooperate during group work

- $\mathrm{c}_{6}$ : present their results and therefore choose suitable technical supplies

- $c_{7}$ : work safely with download services of the Internet

- $\mathrm{c}_{8}$ : explain the function of the Internet

- $\quad c_{9}$ : discuss copyright issues for different scenarios

- $c_{x}: \ldots$

The indirectly addressed and the less often mentioned competencies are left out. Not all of the competencies listed are primary competencies. Some of them are strongly related to the context, like $c_{2}$ and $c_{3}$. Others have a better chance to be found in a similar list for a different context, like $c_{1}, c_{4}$ to $c_{6}$ or $c_{8}$. And some may even last if time and important contexts are changing, like $c_{1}$ or $c_{5}$. The latter are the primary competencies of the context "file sharing" and should be part of an educational standard for informatics.

We know that every step of this simulated analysis opens a wider field for further research. But this approach can be an opportunity not only to generate but also to evaluate existing educational standards if they are suitable for today and the future.

\section{Conclusion}

In this paper, we gave a brief overview on reasons why the debate on competencies and skills has been raised. Also an insight to the development of educational standards has been given. After this we showed a new approach for designing educational standards by using the idea of "Informatics in Context". We also opened the discussion if this way could offer a better and more reliable solution for the daily work of teachers. 
A first sketch on how competencies can be gained by analyzing contexts was shown. Of course, a lot of work will still be necessary to gather a set of interesting contexts which can be useful as blueprints for teachers to develop their own contexts. On the other hand, we will have to identify the important ones from which to generate competencies from the wealth of available contexts. Also, this approach may be used to review existing educational standards for informatics, to decide if and which parts of them are suitable and also to help with their improvement.

\section{References}

1. Brinda, T., Puhlmann, H., Schulte, C.: Bridging ICT and CS - Educational Standards for Computer Science in Lower Secondary Education. In: ITiCSE 2009, Paris, France (2009)

2. Diethelm, I., Hildebrandt, C., Krekeler, L.: Implementation of Computer Science in Context - a research perspective regarding teacher-training. In: 9th Baltic Sea Conference on Computing Education Research, Koli Calling, Koli, Finland (2009)

3. Dörge, C.: Competencies and Skills: Filling old Skins with New Wine. In: Reynolds, N., Turcsányi-Szabó, M. (eds.) KCKS 2010. IFIP AICT, vol. 324, pp. 78-89. Springer, Heidelberg (2010)

4. Gesellschaft für Informatik (GI): Grundsätze und Standards für die Informatik in der Schule (Educational Standards for Informatics in secondary schools). LogIn, No. 150/151 (2008)

5. The IniK Project Group: Informatik im Kontext (Informatics in context) (2009), http: / /www. informatik-im-kontext.de

6. Koubek, J., Schulte, C., Schulze, P., Witten, H.: Informatik im Kontext (IniK) - Ein integratives Unterrichtskonzept für den Informatikunterricht (Informatics in Context - an integrated teaching concept for informatics lessons). Informatik und Schule, Berlin (2009)

7. Mertens, D.: Schlüsselqualifikationen - Thesen zur Schulung für eine moderne Gesellschaft (Key competencies - statements for education for a modern society). Mitteilungen aus der Arbeitsmarkt- und Berufsforschung 7, 36-43 (1974)

8. Mulder, F., van Weert, T.: IFIP/UNESCO's Informatics Curriculum Framework 2000 for Higher Education. SIGCSE Bulletin 33(4) (2001)

9. OECD: The Definition and Selection of Key Competencies - Executive Summary (2005), http://www.deseco.admin.ch/bfs/deseco/en/index/03.html

10. Parchmann, I., Gräsel, C., Baer, A., Nentwig, P., Demuth, R., Ralle, B., the ChiK Projekt Group: "Chemie im Kontext": A symbiotic implementation of a context-based teaching and learning approach. International Journal of Science Education 28(9), 1041-1062 (2006)

11. Waddington, D., Nentwig, P., Schanze, S. (eds.): Making it Comparable: Standards in Science Education, Münster, Waxmann (2007)

12. Weinert, F.E.: Leistungsmessung in Schulen (Assessments in schools). Beltz, Weinheim, Basel, Switzerland (2001) 